\title{
Clinical characteristics and risk factors of fatal patients with COVID-19: a retrospective cohort study in Wuhan, China
}

Meng Jin ${ }^{1 \dagger}$, Zequn Lu ${ }^{2 \dagger}$, Xu Zhang ${ }^{3 \dagger}$, Yanan Wang ${ }^{4 \dagger}$, Jing Wang ${ }^{4 \dagger}$, Yimin Cai ${ }^{2 \dagger}$, Kunming Tian $^{5}$, Zezhong Xiong ${ }^{4}$, Qiang Zhong ${ }^{6}$, Xiao Rann ${ }^{6}$, Chunguang Yang ${ }^{4}$, Xing Zeng ${ }^{4}$, Lu Wang ${ }^{2}$, Yao Li ${ }^{2}$, Shanshan Zhang ${ }^{2}$, Tianyi Dong ${ }^{2}$, Xinying Yue ${ }^{2}$, Heng Li ${ }^{4}$, Bo Liu ${ }^{7}$, Xin Chen ${ }^{7}$, Hongyuan Cui ${ }^{8}$, Jirong $\mathrm{Qi}^{9}$, Haining Fan ${ }^{10}$, Haixia $\mathrm{Li}^{11}$, Xiang-Ping Yang ${ }^{12}$, Zhiquan $\mathrm{Hu}^{4}$, Shaogang Wang ${ }^{4}$, Jun $\mathrm{XiaO}^{4^{*+}}$, Ying Wang ${ }^{13^{*+}}$, Jianbo Tian $^{14^{*+}}$ and Zhihua Wang $^{4^{*}+}$ (1)

\begin{abstract}
Background: The coronavirus disease 2019 (COVID-19) has caused a global pandemic, resulting in considerable mortality. The risk factors, clinical treatments, especially comprehensive risk models for COVID-19 death are urgently warranted.
\end{abstract}

Methods: In this retrospective study, 281 non-survivors and 712 survivors with propensity score matching by age, sex, and comorbidities were enrolled from January 13, 2020 to March 31, 2020.

Results: Higher SOFA, qSOFA, APACHE II and SIRS scores, hypoxia, elevated inflammatory cytokines, multi-organ dysfunction, decreased immune cell subsets, and complications were significantly associated with the higher COVID19 death risk. In addition to traditional predictors for death risk, including APACHE II (AUC $=0.83)$, SIRS (AUC $=0.75)$, SOFA (AUC $=0.70$ ) and qSOFA scores ( $A \cup C=0.61$ ), another four prediction models that included immune cells subsets ( $A \cup C=0.90$ ), multiple organ damage biomarkers ( $A \cup C=0.89$ ), complications ( $A \cup C=0.88$ ) and inflammatoryrelated indexes ( $A \cup C=0.75$ ) were established. Additionally, the predictive accuracy of combining these risk factors ( $A \cup C=0.950$ ) was also significantly higher than that of each risk group alone, which was significant for early clinical management for COVID-19.

Conclusions: The potential risk factors could help to predict the clinical prognosis of COVID-19 patients at an early stage. The combined model might be more suitable for the death risk evaluation of COVID-19.

\footnotetext{
*Correspondence: junxiao_hust@163.com; wangyingxiehe@163.com; tianjb@whu.edu.cn; zhwang_hust@hotmail.com

${ }^{\dagger}$ Meng Jin, Zequn Lu, Xu Zhang, Yanan Wang, Jing Wang, and Yimin Cai have contributed equally as co-first authors

†Jun Xiao, Ying Wang, Jianbo Tian, and Zhihua Wang have contributed equally as co-corresponding authors

${ }^{4}$ Department of Urology, Tongji Hospital, Tongji Medical College, Huazhong University of Science and Technology, No. 1095, Jiefang Ave, Wuhan 430030, Hubei, China

${ }^{13}$ Department of Virology, Wuhan Centers for Disease Prevention and Control, Wuhan, Hubei, China

${ }^{14}$ School of Health Sciences, Wuhan University, Wuhan 430071, China

Full list of author information is available at the end of the article
}

(C) The Author(s) 2021. Open Access This article is licensed under a Creative Commons Attribution 4.0 International License, which permits use, sharing, adaptation, distribution and reproduction in any medium or format, as long as you give appropriate credit to the original author(s) and the source, provide a link to the Creative Commons licence, and indicate if changes were made. The images or other third party material in this article are included in the article's Creative Commons licence, unless indicated otherwise in a credit line to the material. If material is not included in the article's Creative Commons licence and your intended use is not permitted by statutory regulation or exceeds the permitted use, you will need to obtain permission directly from the copyright holder. To view a copy of this licence, visit http://creativecommons.org/licenses/by/4.0/. The Creative Commons Public Domain Dedication waiver (http://creativeco mmons.org/publicdomain/zero/1.0/) applies to the data made available in this article, unless otherwise stated in a credit line to the data. 
Keywords: COVID-19, Death risk, Risk prediction models, Immune cells subsets

\section{Background}

The global spread of coronavirus disease 2019 (COVID19) has become a major global public health issue. The COVID-19 pandemic was sweeping across borders, sickening and killing people in nearly every country. Until January 5, 2021, a total of 84,233,579 cases were confirmed and the death numbers of COVID-19 attained $1,843,293$ [1] currently. Considering the adverse effects of COVID-19 and its high mortality rate, it is urgent for us to understand the risk factors that influence the incidence of death and develop a comprehensive risk model evaluating the death risk of COVID-19.

Recent evidence has shown that the infection caused by severe acute respiratory syndrome coronavirus 2 (SARS-CoV-2) induces clusters of severe and even fatal pneumonia which had high mortality [2]. Several scores have been considered as good evaluation indexes for sepsis, septic shock, multi-organ dysfunction, and even death in patients suffering from bacterial or viral pathogen infection, such as the sequential organ failure assessment (SOFA), quick SOFA (qSOFA), acute physiology, chronic health evaluation II (APACHE II) and systemic inflammatory response syndrome (SIRS) scores [3-8]. Besides, it has been reported that the pro-inflammatory cytokine IL-6 and organ damage biomarkers, such as D-dimer and NT-proBNP were risk factors for death of adults with COVID-19 [6, 9, 10]. Some case series have reported the clinical characteristics or potential risk factors for fatal patients with COVID-19 [6, 9, 11]. However, the sample sizes in most of these reports were limited and detailed information about biochemical biomarkers dynamics, immune cell subsets or pertained treatments of fatal COVID-19 patients have not yet been well described. Most importantly, no researches have been done to develop a comprehensive prediction model integrating these scores in the current, inflammatory indices, immune cells subsets and organ damage risk factors for death risk of COVID-19, which is of great value for better risk stratification, death-related biomarkers identification and clinical treatments strategies-making.

Here we performed a retrospective, observational study in fatal COVID-19 patients and presented the details of patients with definite clinical outcome (death or discharge) from Tongji Hospital, Huazhong University of Science and Technology (HUST), which is the biggest designated hospital for treating severely or critically ill COVID-19 patients in Wuhan, an epicenter of COVID-19 outbreak. The objective of this study was to explore potential risk factors of death for COVID-19 and establish a comprehensive risk model evaluating the death risk of COVID-19, which is of utmost importance for early clinical management and successful establishment of standardized treatment protocols, ultimately curbing the rising fatality rate of COVID-19.

\section{Methods \\ Study design and participants}

This retrospective cohort study was performed in patients with confirmed COVID-19 from Tongji Hospital, Tongji Medical College, HUST, a designated hospital for severely or critically ill COVID-19 patients. All fatal patients with COVID-19 $(N=281)$ were enrolled from January 13 to March 31, 2020. Subsequently, 712 survivors were statistically matched by propensity score matching [12] at an approximate ratio of 3:1 based on age, sex and comorbidities. This study was approved by the Ethics Committee of Tongji Hospital, Tongji Medical College, HUST and granted a waiver of informed consent from study participants.

\section{Data collection}

Epidemiological, clinical, radiological, laboratory, clinical treatments, and clinical outcomes data of all patients with laboratory-confirmed SARS-CoV-2 were obtained with data collection forms from electronic medical records of Tongji Hospital. The researches on other topics $[9,13]$ may obtain some information of non-survivors in Tongji hospital, however, our study collected more information about cases and detailed data. The admission and in-hospital data of these patients were collected, reviewed and verified by a trained team of physicians. Any missing or uncertain records were collected and clarified through communication with involved health-care providers and their families. The detailed and standardized information of demographic data, underlying comorbidities, initial symptoms, vital signs, and chest computed tomographic (CT) were recorded or diagnosed at hospital admission. The complications, treatments, clinical outcomes (survivors and non-survivors) and hospital length of stay were monitored to March 31, 2020, the final data of follow-up. Laboratory examinations including blood routine, immune cells subsets, inflammatory cytokines and biomarkers, blood gas assay, cardiac function test, renal function test, liver function test, pancreatic function test, coagulation test, and metabolism test were detected on the first diagnosed date. The time of followup was defined as the duration from admission to outcomes (survivor/non-survivor) of patients. There were no 
cases lost to follow-up in this study attributed to standardized government managements and close tracking for COVID-19 pandemic.

\section{Definitions}

The detailed definitions of complications including acute respiratory distress syndrome (ARDS), acute pancreatic injury, acute liver damage, acute cardiac injury, disseminated intravascular coagulation (DIC), SOFA, qSOFA, APACHE II and SIRS scores were listed in the Additional file 1: Methods.

\section{Statistical analysis}

Continuous variables were presented as median and interquartile range (IQR) or mean and standard deviation (SD). Categorical variables were expressed as number (\%). For continuous variables, Student's $t$-test was used for normal distributed data, and Mann-Whitney $\mathrm{U}$ non-parameter test was used for non-normal distributed data. The Pearson's $\chi^{2}$ test or Fisher's exact test were applied for categorical variables. Time to events (recovered or death) was defined as the time from hospital admission to events. The univariate Cox regression models were used to determine hazard ratios (HRs) and $95 \%$ confidence intervals (CIs) for the association between individual factors and death risk of COVID19. The multivariate Cox proportional hazards model analysis was used to establish different comprehensive death risk score models including four score systems, biochemical biomarkers, complications, and a combined system integrating all scores. The significant variables from the univariable analysis of the death risk were considered as the candidates. The stepwise regression was used for the selection of the prediction for the model. Each comprehensive risk score model of candidate variables was established according to the following formula: Risk score $(\mathrm{RS})=\sum \beta$ variables $\times$ Expvariables. The $\beta$ variables represented the estimated contribution coefficient of independent candidate variables in the multivariate Cox regression analysis and Expvariables denoted the level of independent candidate variables. According to this formula, the risk scores of each patient of different death risk score prediction models were computed. The combined system was performed based on the integrating risk scores of different comprehensive models. Then we calculated sensitivity, the specificity of different death risk score prediction models with survival, timeROC packages for all analyses in $\mathrm{R}$ version 3.50. Time-dependent receiver operating characteristic (ROC) curves and areas under the curves (AUCs) were used to assess the prognostic accuracy of each model [14]. A two-sided $P$ value $<0.05$ was considered statistically significant.

\section{Results}

Clinical characteristics and laboratory findings of non-survivors and survivors with COVID-19

$281(28.30 \%)$ non-survivors and 712 (71.70\%) survivors with COVID-19 were enrolled in this study. Considering that the age, sex, and comorbidities had been reported to be the common death risk factors of COVID-19, enrolled survivors and non-survivors were statistically matched based on these risk factors. As summarized in Table 1 and Additional file 1: Table S1, the most common symptoms of all patients were fever and cough, non-survivors were more likely to have dyspnea $(50.39 \%$ vs $42.23 \%$, $P=0.026)$ than survivors. Besides, non-survivors tended to have more abnormal vital signs on admission, such as higher temperature, heart rate and respiratory rate.

The abnormalities in chest CT images among these patients were also observed (Table 1 and Fig. 1). The patchy shadows presence was the most typical manifestation of both groups, while pleural thickening (41.38\% vs $26.90 \% ; P=0.018$ ) was more frequently observed in nonsurvivors, compared with survivors. Consistently, blood gas analysis revealed the degree of hypoxia was more evident in non-survivors, which presented lower concentration of $\mathrm{PaO}_{2}, \mathrm{SaO}_{2}, \mathrm{PaCO}_{2}, \mathrm{CtCO}_{2}$ and bicarbonate than survivors. These findings suggest more serious impairment of lung function in non-survivors.

Previous studies reported that cytokine storm and lymphopenia were common features in severe COVID19 patients $[2,15]$. We observed that the serum levels of inflammatory cytokines including IL6 (61.35 vs $6.85 \mathrm{pg} /$ $\mathrm{mL} ; P<0.0001)$, IL10 (10.30 vs $5.00 \mathrm{pg} / \mathrm{mL} ; P<0.0001)$, IL8 (28.40 vs $11.60 \mathrm{pg} / \mathrm{mL} ; P<0.0001)$, TNF- $\alpha(11.45$ vs $8.10 \mathrm{pg} / \mathrm{mL} ; \quad P<0.0001)$, IL- $1 \beta(8.15$ vs $6.49 \mathrm{pg} /$ $\mathrm{mL} ; P<0.0001)$ and IL2R $(1148.00$ vs $599.50 \mathrm{U} / \mathrm{mL}$; $P<0.0001)$ were significantly elevated in non-survivors compared with survivors. Moreover, the infection-related biomarkers including ferritin, CRP and procalcitonin also exhibited higher levels in non-survivors. Conversely, the baseline counts of lymphocytes (0.63 vs $1.09 \times 10^{9} / \mathrm{L}$; $P<0.0001), \mathrm{CD}^{+} \mathrm{CD} 9^{-} \mathrm{T}$ cells $(276.50$ vs $905.00 /$ $\mu \mathrm{L}, P<0.0001), \mathrm{CD}^{+} \mathrm{CD}^{+} \mathrm{T}$ cells $(62.00$ vs $280.00 /$ $\mu \mathrm{L} ; P<0.0001)$, B cells $(73.50$ vs $156.00 / \mu \mathrm{L}, P<0.0001)$, NK cells $(36.50$ vs $209.00 / \mu \mathrm{L}, P<0.0001)$ as well as the total number of $\mathrm{T}$ cells, B cells and NK cells (406.00 vs $1337.00 / \mu \mathrm{L} ; \quad P<0.0001)$ were drastically decreased in non-survivors, compared with survivors. Collectively, these findings demonstrate that aggravated inflammatory responses and severe lymphopenia might be correlated with the poor clinical outcome of COVID-19 patients.

Multiple-organ damage was more pronounced in non-survivors. We observed higher levels of ALT, TBIL, LDH, homocysteine, NT-proBNP, hs-cTnI, CK-MB and lower level of ALB/GLO in non-survivors. Besides, 
Table 1 Demographic, clinical, radiographic, laboratory findings of survivors and non-survivors with COVID-19

\begin{tabular}{|c|c|c|c|c|c|c|}
\hline \multirow[t]{2}{*}{ Indicators } & \multirow{2}{*}{$\begin{array}{l}\text { Total } \\
N=993\end{array}$} & \multicolumn{2}{|c|}{ Survivors } & \multicolumn{2}{|c|}{ Non-survivors } & \multirow[t]{2}{*}{$P$ value } \\
\hline & & $N=712$ & & $N=281$ & & \\
\hline \multicolumn{7}{|l|}{ Characteristics } \\
\hline Age, years & $68(63-74)$ & $N=712$ & $68(63-73)$ & $N=281$ & $69(62-77)$ & 0.100 \\
\hline Sex & & $N=712$ & & $N=281$ & & \\
\hline Male & $655(65.96 \%)$ & & $464(65.17 \%)$ & & $191(67.97 \%)$ & 0.401 \\
\hline Female & $338(34.04 \%)$ & & $248(34.83 \%)$ & & $90(32.03 \%)$ & \\
\hline \multicolumn{7}{|l|}{ Comorbidities } \\
\hline Hypertension & $401(40.38 \%)$ & $N=712$ & $292(41.01 \%)$ & $N=281$ & 109(38.79\%) & 0.521 \\
\hline Diabetes & $174(17.52 \%)$ & $N=712$ & 134(18.82\%) & $N=281$ & $40(14.23 \%)$ & 0.087 \\
\hline Coronary heart disease & 104(10.47\%) & $N=712$ & $72(10.11 \%)$ & $N=281$ & $32(11.39 \%)$ & 0.554 \\
\hline Cerebrovascular disease & 39(3.93\%) & $N=712$ & $27(3.79 \%)$ & $N=281$ & $12(4.27 \%)$ & 0.727 \\
\hline Pulmonary tuberculosis & $22(2.22 \%)$ & $N=712$ & $13(1.83 \%)$ & $N=281$ & $9(3.20 \%)$ & 0.184 \\
\hline Hepatitis & $17(1.71 \%)$ & $N=712$ & $10(1.40 \%)$ & $N=281$ & $7(2.49 \%)$ & 0.234 \\
\hline Chronic bronchitis & 19(1.91\%) & $N=712$ & $11(1.54 \%)$ & $N=281$ & $8(2.85 \%)$ & 0.177 \\
\hline $\begin{array}{l}\text { Chronic obstructive pulmonary } \\
\text { disease }\end{array}$ & $9(0.91 \%)$ & $N=712$ & $5(0.70 \%)$ & $N=281$ & $4(1.42 \%)$ & 0.280 \\
\hline Initial symptoms & $N=919$ & & $N=663$ & & $N=256$ & \\
\hline Fever & 718(78.13\%) & & $523(78.88 \%)$ & & 195(76.17\%) & 0.373 \\
\hline Cough & $632(68.77 \%)$ & & $471(71.04 \%)$ & & $161(62.89 \%)$ & $0.017^{*}$ \\
\hline Dyspnea & $409(44.50 \%)$ & & $280(42.23 \%)$ & & 129(50.39\%) & $0.026^{*}$ \\
\hline Expectoration & $399(43.42 \%)$ & & 293(44.19\%) & & 106(41.41\%) & 0.445 \\
\hline Diarrhoea & 199(21.65\%) & & $140(21.12 \%)$ & & $59(23.05 \%)$ & 0.524 \\
\hline Fatigue & $170(18.50 \%)$ & & 119(17.95\%) & & $51(19.92 \%)$ & 0.490 \\
\hline Chest tightness & $147(16.00 \%)$ & & 106(15.99\%) & & $41(16.02 \%)$ & 0.992 \\
\hline Chills & $97(10.55 \%)$ & & $72(10.86 \%)$ & & $25(9.77 \%)$ & 0.628 \\
\hline Myalgia & $82(8.92 \%)$ & & $67(10.11 \%)$ & & 15(5.86\%) & $0.043^{*}$ \\
\hline Anorexia & $71(7.73 \%)$ & & $50(7.54 \%)$ & & $21(8.20 \%)$ & 0.736 \\
\hline Headache & $56(6.09 \%)$ & & $43(6.49 \%)$ & & $13(5.08 \%)$ & 0.424 \\
\hline Vertigo & $40(4.35 \%)$ & & $21(3.17 \%)$ & & 19(7.42\%) & $0.005^{*}$ \\
\hline Others ${ }^{n}$ & $124(13.49 \%)$ & & 104(15.69\%) & & $20(7.81 \%)$ & $0.002^{*}$ \\
\hline \multicolumn{7}{|l|}{ Vital signs } \\
\hline Temperature, ${ }^{\circ} \mathrm{C}$ & $36.6(36.3-37.1)$ & $N=712$ & $36.5(36.2-36.9)$ & $N=281$ & $36.8(36.4-37.6)$ & $<0.0001^{*}$ \\
\hline Heart rate, bpm & $92(80-105)$ & $N=712$ & 88(78-99) & $N=281$ & 102(90-115) & $<0.0001^{*}$ \\
\hline Respiratory rate, bpm & $20(20-23)$ & $N=712$ & $20(20-21)$ & $N=281$ & $26(21-33)$ & $<0.0001^{*}$ \\
\hline Mean arterial pressure, $\mathrm{mmHg}$ & $95(86.67-104.33)$ & $N=711$ & $96.67(89.33-105.67)$ & $N=281$ & 88.67(79-99) & $<0.0001^{*}$ \\
\hline $\mathrm{CT}$ findings & $N=757$ & & $N=699$ & & $N=58$ & \\
\hline Patchy shadows & $586(77.41 \%)$ & & $544(77.83 \%)$ & & $42(72.41 \%)$ & 0.344 \\
\hline Ground-glass opacity & $338(44.65 \%)$ & & $307(43.92 \%)$ & & $31(53.45 \%)$ & 0.161 \\
\hline Fibrous stripes & $262(34.61 \%)$ & & $253(36.19 \%)$ & & $9(15.52 \%)$ & $0.001^{*}$ \\
\hline Pleural thickening & $212(28.01 \%)$ & & $188(26.90 \%)$ & & $24(41.38 \%)$ & $0.018^{*}$ \\
\hline Nodules & $60(7.93 \%)$ & & $55(7.87 \%)$ & & $5(8.62 \%)$ & $0.800^{\mathrm{a}}$ \\
\hline Lymphadenia & $214(28.27 \%)$ & & $192(27.47 \%)$ & & $22(37.93 \%)$ & 0.089 \\
\hline Bilateral pulmonary & $735(97.09 \%)$ & & $677(96.85 \%)$ & & $58(100.00 \%)$ & $0.413^{\mathrm{a}}$ \\
\hline Right lung & $12(1.59 \%)$ & & $12(1.72 \%)$ & & $0(0.00 \%)$ & $0.614^{\mathrm{a}}$ \\
\hline Left lung & $10(1.32 \%)$ & & $10(1.43 \%)$ & & $0(0.00 \%)$ & $1.000^{\mathrm{a}}$ \\
\hline \multicolumn{7}{|l|}{ Laboratory examinations } \\
\hline \multicolumn{7}{|l|}{ Blood routine } \\
\hline Leukocytes, \# $(N=993), \times 10^{9} / \mathrm{L}$ & $6.12(4.66-8.28)$ & $N=712$ & $5.63(4.43-7.14)$ & $N=281$ & $8.91(6.00-13.03)$ & $<0.0001^{*}$ \\
\hline Monocytes, \% $(N=993)$ & $8.10(5.40-10.40)$ & $N=712$ & $8.90(7.00-10.90)$ & $N=281$ & $4.60(2.70-7.50)$ & $<0.0001^{*}$ \\
\hline
\end{tabular}


Table 1 (continued)

\begin{tabular}{|c|c|c|c|c|c|c|}
\hline \multirow[t]{2}{*}{ Indicators } & \multirow{2}{*}{$\begin{array}{l}\text { Total } \\
N=993\end{array}$} & \multicolumn{2}{|c|}{ Survivors } & \multicolumn{2}{|c|}{ Non-survivors } & \multirow[t]{2}{*}{$P$ value } \\
\hline & & $N=712$ & & $N=281$ & & \\
\hline Neutrophils, \% $(N=993)$ & $73.60(63.70-83.90)$ & $N=712$ & $69.00(60.30-77.35)$ & $N=281$ & $87.10(79.90-92.20)$ & $<0.0001^{*}$ \\
\hline Eosinophils, \% (N=993) & $0.30(0.00-1.30)$ & $N=712$ & $0.70(0.00-1.70)$ & $N=281$ & $0.00(0.00-0.10)$ & $<0.0001^{*}$ \\
\hline \multicolumn{7}{|l|}{ Immune cell subsets } \\
\hline Lymphocytes, \# $(\mathrm{N}=993), \times 10^{9} / \mathrm{L}$ & $0.94(0.64-1.34)$ & $N=712$ & $1.09(0.76-1.46)$ & $N=281$ & $0.63(0.44-0.85)$ & $<0.0001^{*}$ \\
\hline $\mathrm{CD}^{+}{ }^{+} \mathrm{CD} 19^{-} \mathrm{T}$ cells, $\#(N=207), / \mu \mathrm{L}$ & $782.00(394.00-1060.00)$ & $N=153$ & $905.00(682.00-1173.00)$ & $N=54$ & $276.50(132.75-408.50)$ & $<0.0001^{*}$ \\
\hline $\mathrm{CD}^{+} \mathrm{CD}^{+} \mathrm{T}$ cells, $\#(N=207), / \mu \mathrm{L}$ & $248.00(112.50-350.50)$ & $N=153$ & $280.00(206.00-384.00)$ & $N=54$ & $62.00(29.25-127.00)$ & $<0.0001^{*}$ \\
\hline $\mathrm{CD}^{-}{ }^{-} \mathrm{CD}_{19}{ }^{+} \mathrm{B}$ cells, $\#(N=207), / \mu \mathrm{L}$ & $137.00(78.00-205.00)$ & $N=153$ & $156.00(117.00-209.00)$ & $N=54$ & $73.50(40.25-143.00)$ & $<0.0001^{*}$ \\
\hline $\begin{array}{l}\mathrm{CD}^{-}{ }^{-} \mathrm{CD} 16^{+} \mathrm{CD}^{2} 6^{+} \mathrm{NK} \text { cells, \# } \\
(\mathrm{N}=207), / \mu \mathrm{L}\end{array}$ & $160.00(72.50-278.50)$ & $N=153$ & $209.00(128.00-313.00)$ & $N=54$ & $36.50(16.00-74.75)$ & $<0.0001^{*}$ \\
\hline $\begin{array}{l}\text { T cells + B cells + NK cells, \# } \\
(N=207), / \mu \mathrm{L}\end{array}$ & 1134.00(687.00-1533.00) & $N=153$ & 1337.00(995.00-1662.00) & $N=54$ & $406.00(269.75-692.50)$ & $<0.0001^{*}$ \\
\hline \multicolumn{7}{|l|}{$\begin{array}{l}\text { Inflammatory cytokines and bio- } \\
\text { markers }\end{array}$} \\
\hline IL-6 (N=796), pg/mL & 14(3.42-49.38) & $N=594$ & $6.85(2.82-24.18)$ & $N=202$ & $61.35(29.27-151.45)$ & $<0.0001^{*}$ \\
\hline $\mathrm{IL}-10(\mathrm{~N}=778), \mathrm{pg} / \mathrm{mL}$ & $5.00(5.00-8.90)$ & $N=579$ & $5.00(5.00-5.85)$ & $N=199$ & 10.30(6.35-18.70) & $<0.0001^{*}$ \\
\hline IL-8 (N=779), pg/mL & $14.20(8.00-27.95)$ & $N=579$ & $11.60(6.80-20.25)$ & $N=200$ & $28.40(16.35-61.83)$ & $<0.0001^{*}$ \\
\hline TNF-a $(N=786), \mathrm{pg} / \mathrm{mL}$ & $8.60(6.60-11.80)$ & $N=586$ & $8.10(6.10-10.70)$ & $N=200$ & $11.45(8.1-16.50)$ & $<0.0001^{*}$ \\
\hline $\mathrm{IL}-1 \beta(N=778), \mathrm{pg} / \mathrm{mL}$ & $6.92(7.44)$ & $N=578$ & $6.49(6.35)$ & $N=200$ & $8.15(9.88)$ & $<0.0001^{*}$ \\
\hline $\mathrm{IL}-2 \mathrm{R}(\mathrm{N}=774), \mathrm{U} / \mathrm{mL}$ & $675.50(446.25-1078.00)$ & $N=576$ & $599.50(407.75-873.25)$ & $N=198$ & $1148.00(740.25-1615.00)$ & $<0.0001^{*}$ \\
\hline Ferritin $(N=602), \mu \mathrm{g} / \mathrm{L}$ & 751.00(421.10-1439.05) & $N=420$ & $566.90(351.53-989.13)$ & $N=182$ & 1407.35(832.68-2400.18) & $<0.0001^{*}$ \\
\hline hs-CRP $(N=980), \mathrm{mg} / \mathrm{L}$ & $41.05(6.90-98.38)$ & $N=704$ & 20.85(3.58-66.33) & $N=276$ & 105.60(59.33-164.43) & $<0.0001^{*}$ \\
\hline Procalcitonin $(N=890), \mathrm{ng} / \mathrm{mL}$ & $0.08(0.05-0.21)$ & $N=629$ & $0.06(0.04-0.09)$ & $N=261$ & $0.29(0.12-0.89)$ & $<0.0001^{*}$ \\
\hline \multicolumn{7}{|l|}{ Organ damage indexes } \\
\hline $\operatorname{ALT}(N=991), \mathrm{U} / \mathrm{L}$ & $24.00(15.00-39.00)$ & $N=710$ & $23.00(15.00-38.00)$ & $N=281$ & $27.00(18.00-42.00)$ & $0.004^{*}$ \\
\hline $\operatorname{AST}(N=991), U / L$ & $30.00(21.00-44.00)$ & $N=710$ & $26.50(19.00-38.00)$ & $N=281$ & $41.00(29.00-59.00)$ & $<0.0001^{*}$ \\
\hline TBIL $(N=993), \mu \mathrm{mol} / \mathrm{L}$ & 10.00(7.40-13.90) & $N=712$ & $9.40(7.20-12.55)$ & $N=281$ & $12.30(9.00-18.60)$ & $<0.0001^{*}$ \\
\hline ALB/GLO $(N=988)$ & $0.99(0.83-1.21)$ & $N=708$ & $1.05(0.89-1.26)$ & $N=280$ & $0.86(0.75-1.00)$ & $<0.0001^{*}$ \\
\hline $\mathrm{LDH}(N=950), \mathrm{U} / \mathrm{L}$ & $302.00(225.00-442.75)$ & $N=673$ & $262.00(209.00-328.00)$ & $N=277$ & $504.00(364.00-669.00)$ & $<0.0001^{*}$ \\
\hline $\operatorname{ALP}(N=952), U / L$ & $68.00(56.00-86.00)$ & $N=671$ & $66.00(55.50-79.00)$ & $N=281$ & $78.00(60.00-107.00)$ & $<0.0001^{*}$ \\
\hline Amylase $(N=486), \mathrm{U} / \mathrm{L}$ & $63.50(43.00-83.00)$ & $N=342$ & $67.00(48.00-83.00)$ & $N=144$ & $52.00(36.00-84.00)$ & $0.004^{*}$ \\
\hline $\operatorname{eGFR}(N=987), \mathrm{mL} /\left(\mathrm{min}^{*} 1.73 \mathrm{~m}^{2}\right)$ & $82.60(66.80-92.80)$ & $N=709$ & $85.90(71.60-93.00)$ & $N=278$ & $72.60(47.83-90.98)$ & $<0.0001^{*}$ \\
\hline Creatinine $(N=988), \mu \mathrm{mol} / \mathrm{L}$ & $78.00(65.00-93.25)$ & $N=712$ & $75.00(64.00-88.00)$ & $N=281$ & $86.00(66.50-114.00)$ & $<0.0001^{*}$ \\
\hline NT-proBNP $(N=841), \mathrm{pg} / \mathrm{mL}$ & $230.00(89.00-744.00)$ & $N=589$ & $152.00(64.00-335.00)$ & $N=252$ & $888.50(362.50-2567.00)$ & $<0.0001^{*}$ \\
\hline CK-MB $(N=650), \mathrm{U} / \mathrm{L}$ & $1.00(0.60-1.90)$ & $N=477$ & $0.80(0.50-1.30)$ & $N=173$ & $2.40(1.20-5.80)$ & $<0.0001^{*}$ \\
\hline hs-cTnl $(N=905), \mathrm{pg} / \mathrm{mL}$ & $7.70(3.60-20.70)$ & $N=644$ & $5.40(2.80-10.93)$ & $N=261$ & $35.30(11.20-194.70)$ & $<0.0001^{*}$ \\
\hline Platelets, \# $(N=988), \times 10^{9} / \mathrm{L}$ & $206.00(149.00-273.25)$ & $N=712$ & $219.00(167.00-285.00)$ & $N=281$ & $159.00(111.00-223.50)$ & $<0.0001^{*}$ \\
\hline $\mathrm{PT}(N=980), \mathrm{s}$ & $14.10(13.50-15.00)$ & $N=699$ & $13.90(13.30-14.40)$ & $N=281$ & $15.30(14.30-16.90)$ & $<0.0001^{*}$ \\
\hline $\operatorname{APTT}(N=930), s$ & $39.40(36.10-43.50)$ & $N=680$ & $39.25(36.00-42.83)$ & $N=250$ & $40.25(36.23-46.00)$ & $0.005^{*}$ \\
\hline D-Dimer $(N=966), \mu \mathrm{g} / \mathrm{mL}$ & $1.14(0.51-2.68)$ & $N=691$ & $0.77(0.41-1.64)$ & $N=275$ & $4.10(1.39-15.70)$ & $<0.0001^{*}$ \\
\hline $\operatorname{FDP}(N=727), g / L$ & $4.50(4.00-14.40)$ & $N=515$ & $4.00(4.00-5.50)$ & $N=212$ & 25.85(7.40-86.50) & $<0.0001^{*}$ \\
\hline PTA $(N=980), \%$ & $87.00(77.75-95.25)$ & $N=699$ & $90.00(84.00-98.00)$ & $N=281$ & $75.00(62.00-86.00)$ & $<0.0001^{*}$ \\
\hline \multicolumn{7}{|l|}{ Metabolism indexes } \\
\hline $\operatorname{HbA1c}(N=351), \%$ & $6.40(6.00-7.30)$ & $N=251$ & $6.30(6.00-7.15)$ & $N=100$ & $6.65(6.10-7.43)$ & $0.022^{*}$ \\
\hline $\mathrm{K}^{+}(\mathrm{N}=993), \mathrm{mmol} / \mathrm{L}$ & $4.23(3.80-4.61)$ & $N=712$ & $4.19(3.78-4.55)$ & $N=281$ & $4.36(3.88-4.86)$ & $0.001^{*}$ \\
\hline $\mathrm{Ca}^{2+}(\mathrm{N}=992), \mathrm{mmol} / \mathrm{L}$ & $2.12(2.04-2.21)$ & $N=712$ & $2.15(2.07-2.23)$ & $N=281$ & 2.06(1.99-2.14) & $<0.0001^{*}$ \\
\hline \multicolumn{7}{|l|}{ Blood gas characteristics } \\
\hline $\mathrm{PaO}_{2}(\mathrm{~N}=303), \mathrm{mmHg}$ & $82.00(68.00-88.00)$ & $N=181$ & $85.70(81.00-92.00)$ & $N=122$ & $68.30(52.30-80.80)$ & $<0.0001^{*}$ \\
\hline $\mathrm{SaO}_{2}(N=303), \%$ & $95.70(88.00-98.50)$ & $N=181$ & $98.10(95.90-99.10)$ & $N=122$ & $85.50(71.00-92.00)$ & $<0.0001^{*}$ \\
\hline $\mathrm{PaCO}_{2}(\mathrm{~N}=304), \mathrm{mmHg}$ & $37.50(32.28-42.33)$ & $N=182$ & $39.80(34.50-43.35)$ & $N=122$ & $34.60(30.30-37.90)$ & $<0.0001^{*}$ \\
\hline
\end{tabular}


Table 1 (continued)

\begin{tabular}{|c|c|c|c|c|c|c|}
\hline \multirow[t]{2}{*}{ Indicators } & \multirow{2}{*}{$\begin{array}{l}\text { Total } \\
N=993\end{array}$} & \multicolumn{2}{|c|}{ Survivors } & \multicolumn{2}{|c|}{ Non-survivors } & \multirow[t]{2}{*}{$P$ value } \\
\hline & & $N=712$ & & $N=281$ & & \\
\hline $\begin{array}{l}\text { Actual bicarbonate }(N=304) \text {, } \\
\mathrm{mmol} / \mathrm{L}\end{array}$ & $23.75(20.50-25.80)$ & $N=182$ & $24.40(22.25-26.15)$ & $N=122$ & $22.30(19.30-24.90)$ & $<0.0001^{*}$ \\
\hline Score prediction & $N=303$ & & $N=181$ & & $N=122$ & \\
\hline SOFA score & $3.00(2.00-4.00)$ & & $2.00(2.00-3.00)$ & & $4.00(3.00-6.00)$ & $<0.0001^{*}$ \\
\hline qSOFA score & $0.00(0.00-1.00)$ & & $0.00(0.00-1.00)$ & & $1.00(0.25-1.00)$ & $<0.0001^{*}$ \\
\hline APACHE II score & 13.00(9.00-17.00) & & 10.00(7.00-13.00) & & $17.00(14.00-20.00)$ & $<0.0001^{*}$ \\
\hline SIRS score & $1.00(1.00-2.00)$ & & $1.00(0.00-2.00)$ & & $2.00(1.00-2.00)$ & $<0.0001^{*}$ \\
\hline
\end{tabular}
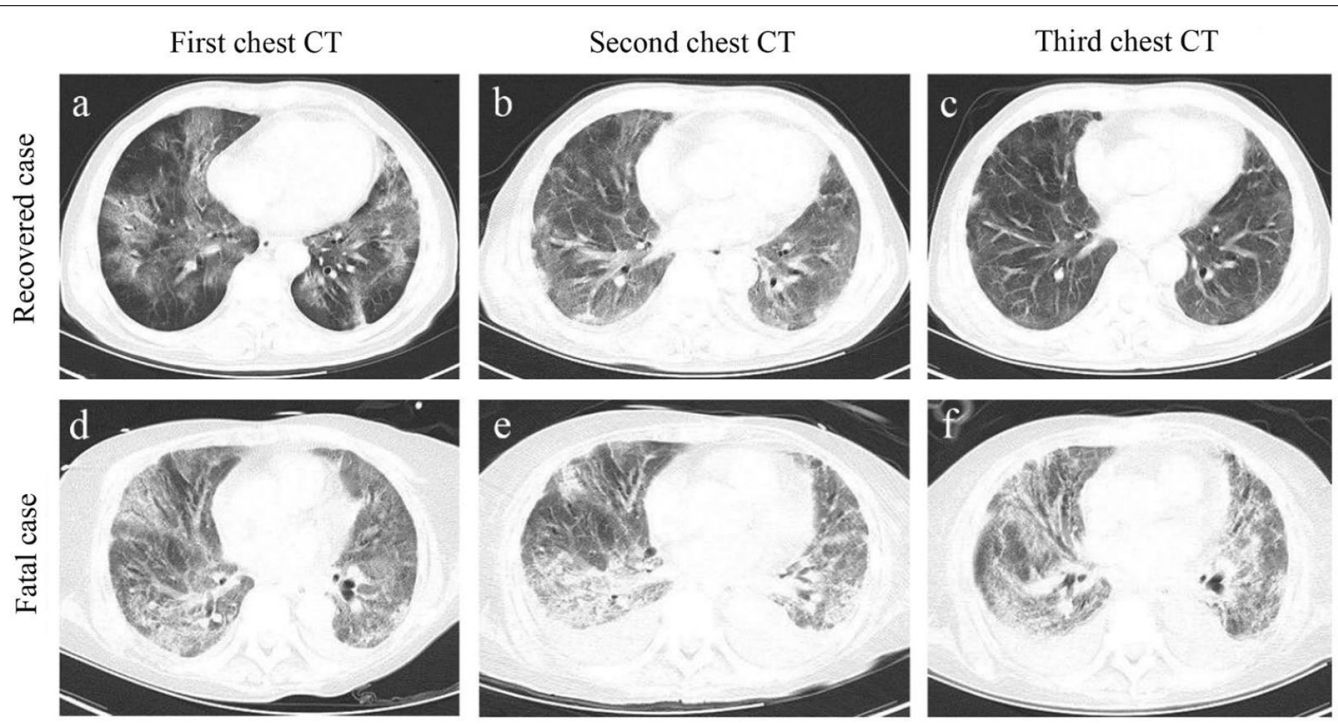

Fig. 1 Representative chest computed tomographic images of fatal and recovered patients with COVID-19. a-c. An elderly male recovered case confirmed with COVID-19 at different disease stages. a Axial chest CT image obtained at the onset shows diffuse ground-glass opacity (GGO) and fibrous stripes; $\mathbf{b}$ axial chest CT image obtained at the middle stage shows bilateral, peripheral GGO and fibrous stripes associated with smooth interlobular and intralobular septal thickening; $\mathbf{c}$ axial chest CT image obtained at discharge stage shows bilateral fibrous stripes and nodules. $\mathbf{d}-\mathbf{f}$ An elderly male fatal case confirmed with COVID-19 at different disease stages. a Axial chest CT obtained at the onset shows bilateral diffuse GGO associated with round cystic change, local bronchial meteorology and left pleural effusion; e axial chest CT image obtained at the middle stage shows the progression of bilateral GGO, bronchial meteorology and pleural effusion; $\mathbf{f}$ axial chest CT image obtained near stage of death shows the progression of bilateral GGO, round cystic change, bronchial meteorology, and increase of pleural effusion

non-survivors had a falling count of eosinophils while elevated leukocytes and neutrophils, compared with survivors. Of note, in non-survivors, coagulation-related biomarkers of platelets counts were also substantially decreased, followed by the increased D-dimer and prolonged PT and APTT.

Additionally, in term of several scores evaluating disease severity, the fatal cases had higher SOFA (4.00 vs $2.00, P<0.0001)$, qSOFA (1.00 vs $0.00, P<0.0001)$, APACHE II $(17.00$ vs $10.00, P<0.0001)$ and SIRS scores (2.00 vs $1.00, P<0.0001$ ), compared to recovered patients (Table 1).

\section{Complications and clinical treatments of non-survivors and survivors with COVID-19}

SARS-COV-2 infection can cause both pulmonary and multi-system inflammation, leading critical complications (Table 2). The frequency of acute cardiac injury $(79.72 \%$ vs $11.80 \%, P<0.0001)$, heart failure $(71.53 \%$ vs $6.32 \%, P<0.0001)$, acute kidney injury $(48.40 \%$ vs $4.35 \%, P<0.0001)$, acute liver injury $(24.20 \%$ vs $2.39 \%$, $P<0.0001)$, acute pancreatic injury $(4.27 \%$ vs $1.54 \%$, $P=0.010)$ especially acute respiratory distress syndrome (ARDS, $96.80 \%$ vs $53.79 \%, P<0.0001$ ) and disseminated intravascular coagulation (DIC, $19.22 \%$ vs 
Table 2 Complications and clinical treatments of survivors and non-survivors with COVID-19

\begin{tabular}{|c|c|c|c|c|}
\hline Indicators & $\begin{array}{l}\text { Total } \\
N=993\end{array}$ & $\begin{array}{l}\text { Survivors } \\
N=712\end{array}$ & $\begin{array}{l}\text { Non-survivors } \\
N=\mathbf{2 8 1}\end{array}$ & $P$ value \\
\hline \multicolumn{5}{|l|}{ Complications } \\
\hline ARDS & $655(65.96 \%)$ & $383(53.79 \%)$ & 272(96.80\%) & $<0.0001^{*}$ \\
\hline Acute cardiac injury & $308(31.02 \%)$ & $84(11.80 \%)$ & 224(79.72\%) & $<0.0001^{*}$ \\
\hline Heart failure & $246(24.77 \%)$ & $45(6.32 \%)$ & $201(71.53 \%)$ & $<0.0001^{*}$ \\
\hline Acute kidney injury & $167(16.82 \%)$ & $31(4.35 \%)$ & $136(48.40 \%)$ & $<0.0001^{*}$ \\
\hline Hyperkalaemia & $150(15.11 \%)$ & $38(5.34 \%)$ & 112(39.86\%) & $<0.0001^{*}$ \\
\hline Acidosis & $105(10.57 \%)$ & $42(5.90 \%)$ & $63(22.42 \%)$ & $<0.0001^{*}$ \\
\hline Acute liver injury & $85(8.56 \%)$ & $17(2.39 \%)$ & $68(24.20 \%)$ & $<0.0001^{*}$ \\
\hline Alkalosis & $67(6.75 \%)$ & $44(6.18 \%)$ & $23(8.19 \%)$ & 0.256 \\
\hline DIC & $58(5.84 \%)$ & $4(0.56 \%)$ & $54(19.22 \%)$ & $<0.0001^{*}$ \\
\hline Acute pancreatic injury & $23(2.32 \%)$ & $11(1.54 \%)$ & $12(4.27 \%)$ & $0.010^{*}$ \\
\hline Length of hospital stay & $18(10-26)$ & $22(15-28)$ & $8(4-14)$ & $<0.0001^{*}$ \\
\hline \multicolumn{5}{|l|}{ ICU admission } \\
\hline ICU & $151(15.21 \%)$ & $4(0.56 \%)$ & $147(52.69 \%)$ & $<0.0001^{*}$ \\
\hline Non-ICU & $842(84.79 \%)$ & 708(99.44\%) & 134(47.31\%) & \\
\hline \multicolumn{5}{|l|}{ Treatment $^{\mathrm{a}}$} \\
\hline Antibiotics & $894(90.03 \%)$ & $630(88.48 \%)$ & 264(93.95\%) & $0.0094^{*}$ \\
\hline Glucocorticoid therapy & $754(75.93 \%)$ & $502(70.51 \%)$ & $252(89.68 \%)$ & $<0.0001^{*}$ \\
\hline Antiviral therapy & $874(88.02 \%)$ & 649(91.15\%) & $225(80.07 \%)$ & $<0.0001^{*}$ \\
\hline Intravenous immunoglobulin therapy & $338(34.04 \%)$ & $227(31.88 \%)$ & $101(35.94 \%)$ & $<0.0001^{*}$ \\
\hline Transfusion & 99(9.97\%) & $21(2.95 \%)$ & $78(27.76 \%)$ & $<0.0001^{*}$ \\
\hline Interferon inhalation & $353(35.55 \%)$ & $298(41.85 \%)$ & $55(19.57 \%)$ & $<0.0001^{*}$ \\
\hline High flow nasal cannula & $241(24.27 \%)$ & $43(6.04 \%)$ & 198(70.46\%) & $<0.0001^{*}$ \\
\hline Mechanical ventilation & $365(36.76 \%)$ & $122(17.13 \%)$ & $243(86.48 \%)$ & $<0.0001^{*}$ \\
\hline Non-invasive & $311(31.32 \%)$ & 119(16.71\%) & 192(68.33\%) & $<0.0001^{*}$ \\
\hline Invasive & $54(5.44 \%)$ & $3(0.42 \%)$ & $51(18.15 \%)$ & $<0.0001^{*}$ \\
\hline Continuous renal replacement therapy & $12(12.08 \%)$ & $2(0.28 \%)$ & $10(3.56 \%)$ & $<0.0001^{*}$ \\
\hline Extracorporeal membrane oxygenation & $7(0.70 \%)$ & $1(0.14 \%)$ & $6(2.14 \%)$ & $0.0026^{*}$ \\
\hline
\end{tabular}

COVID-19 Coronavirus disease 2019; ARDS Acute respiratory distress syndrome; DIC Disseminated intravascular coagulation; ICU Intensive care unit

${ }^{a}$ Treatments include antibiotics (cephalosporin, fluoroquinolones, macrolides), antiviral therapy (Lopinavir/ritonavir, ganciclovir, Riboviron, oseltamivir), transfusion (suspended red blood cells, platelets, plasma)

Categorical variables were expressed as number (\%). $P$ values were calculated by Pearson $\chi^{2}$ test, or Fisher's exact test(a). ${ }^{*} P<0.05$

$0.56 \%, P<0.0001)$ were more higher in non-survivors than survivors. These critical complications could be the main cause of death, and the underlying mechanisms warrant further investigations.

Almost all non-survivors received antibiotic treatment $(93.95 \%$ vs $88.48 \%, P=0.0094)$, more than the number of recovered patients (Table 2). Fatal patients received more glucocorticoid therapy, intravenous immunoglobulin therapy or transfusion than recovered cases, since the cytokine storm and DIC were more often observed in non-survivors. Similarly, due to the higher proportions of patients developed acute kidney injury or ARDS in non-survivors, deceased patients received more mechanical ventilation, continuous renal replacement therapy, high flow nasal cannula and extracorporeal membrane oxygenation than recovered patients. These factors might result in more frequent ICU admission $(52.69 \%$ vs $0.56 \%, P<0.0001)$ and shorter time of hospital stay (22[IQR 15-28] vs 8 [IQR $4-14], P<0.0001)$ in non-survivors than survivors. The clinical interventions were more intensive in non-survivors due to the more severe illness in fatal cases. Of note, recovered patients were undergoing more, antiviral therapy $(91.15 \%$ vs $80.07 \%, P<0.0001)$ as compared to decreased cases.

\section{Risk factors associated with the death of COVID-19 patients}

Furthermore, we performed Cox analysis to identify the potential death risk factors of COVID-19 
with adjustment of age, sex, comorbidities. As shown in Table 3 and Additional file 1: Table S2, the higher temperature, faster heart rate and respiratory rate and lower mean arterial pressure were associated with increased risk of death. In terms of laboratory parameters, elevated inflammatory cytokines and infectionrelated factors, such as TNF- $\alpha$, IL-6, IL-10, IL-8, IL-1 $\beta$, IL-2R, ferritin, hs-CRP and procalcitonin were significantly associated with higher death risk of COVID-19. Conversely, the immune cells subsets, such as lymphocytes (HR 0.220, 95\% CI 0.161-0.302, $P<0.0001$ ), B cells (HR 0.994, 95\% CI 0.990-0.998, $P=0.002$ ), NK cells (HR 0.985, 95\% CI 0.981-0.990, $P<0.0001$ ), $\mathrm{CD}^{+}{ }^{+} \mathrm{CD} 19^{-} \mathrm{T}$ cells (HR 0.996, 95\% CI 0.995-0.997, $P<0.0001)$ and $\mathrm{CD}^{+}{ }^{+} \mathrm{CD} 8^{+} \mathrm{T}$ cells (HR $0.989,95 \% \mathrm{CI}$ $0.985-0.992, P<0.0001)$, were significantly associated with the lower death risk of COVID-19.

Indicators that represented organ damages, such as elevated ALT, AST, LDH, creatinine, amylase, hscTnI, NT-proBNP, CK-MB, and decreased ALB/ GLO aggrandized the risk for COVID-19 death. Furthermore, coagulation-related biomarkers, including declined platelet counts (HR 0.994, 95\% CI 0.9920.995, $P<0.0001$ ), and increased PT, APTT, D-Dimer (HR 1.025-1.359, $P<0.0001$ ), might be strong indicators for death risk of COVID-19. Moreover, apart from abnormal biochemical dynamics, metabolism indices and blood gas analysis such as decreased serum level of $\mathrm{PaO}_{2}, \mathrm{SaO}_{2}, \mathrm{PaCO}_{2}$, and $\mathrm{CtCO}_{2}$ could result in the degree of electrolyte disturbance or hypoxia, increasing the risk of death of COVID-19.

We also analyzed the risk of death for patients with complications, patients with ARDS (HR 16.216, 95\% CI 8.341-31.527, $P<0.0001$ ) had highest risk of death, followed by acute cardiac injury (HR 13.023, 95\% CI 9.678-17.524, $P<0.0001$ ), heart failure (HR 10.722, 95\% CI 8.227-13.974, $P<0.0001$ ), acute kidney injury (HR 6.063, 95\% CI 4.759-7.724, $P<0.0001$ ) and DIC (HR 5.819, 95\% CI 4.281-7.910, $P<0.0001$ ). Besides, the higher levels of SOFA, qSOFA, APACHE II and SIRS scores were significantly associated with increased death risk (HR 1.195-3.471, $P<0.0001$ ). These findings provided evidence supporting that dynamic of inflammatory cytokines, immune cells subsets, blood gas, organ damage biomarkers, and especially complications should be closely monitored, in case of poor outcomes.

\section{Comprehensive prediction models for death risk of COVID-19 patients}

In light of the SOFA, qSOFA, APACHE II and SIRS scores have been reported as good diagnostic indicators for sepsis, septic shock and multi-organ failure [3,
$4,6-8,16]$, we then calculated the prediction accuracy of these four scores in assessing death risk of COVID19. As presented in Fig. 2, these four scores had prominent prediction capacities evaluating COVID-19 death risk. The AUCs of SOFA, qSOFA, APACHE II and SIRS scores attained $0.697(95 \%$ CI $0.546-0.849), 0.610(95 \%$ CI $0.474-0.747), 0.826(95 \%$ CI $0.671-0.981)$ and $0.749(95 \%$ CI 0.629-0.869). Of note, discrimination of death risk models were better using APACHE II (AUC $=0.826$, $P=0.022)$ and SIRS scores (AUC $=0.749, P=0.013$ ) than SOFA or qSOFA scores, which might partly be attributed to aggravated pro-inflammatory responses in non-survivors.

Besides, we also established another four prediction models based on inflammatory-related indices, immune cells subsets, organ damage biomarkers and complications, all of which were significantly associated with the COVID-19 death risk. Among death risk prediction models of each group alone, the predictive accuracy of the immune cells subsets group was the highest (AUC $=0.901,95 \%$ CI 0.801-1.000). Similarly, multipleorgan damage biomarkers (AUC $=0.894,95 \%$ CI 0.829 0.959), inflammatory-related indices (AUC $=0.757,95 \%$ CI $0.665-0.850$ ) and complications (AUC $=0.878,95 \%$ CI $0.817-0.938$ ) had better predictive effects in the discrimination of mortality, outperforming abovementioned SOFA, qSOFA scores $(P<0.05)$ (Fig. 2).

Finally, we integrated four score predictive systems, inflammatory-related indices, immune cells subsets, organ damage biomarkers and complications to construct a combined group. The combined score $(\mathrm{AUC}=0.950$, 95\% CI 0.853-1.000) was significantly higher than that of each risk group alone (Fig. 2), suggesting the combined score system can comprehensively reflect the death risk of COVID-19.

\section{Discussion}

This retrospective large cohort study identified several risk factors and established comprehensive risk models for death in COVID-19 patients. In particular, patients with higher SOFA, qSOFA, APACHE II and SIRS scores, decreased immune cells subsets, elevated inflammatoryrelated indices, dysregulated multi-organ damage biomarkers and deleterious complications had a higher risk of in-hospital death of COVID-19. Notably, the predictive accuracy of the immune cells subsets group was the highest (AUC $=0.901$ ) among groups alone. Additionally, the predictive accuracy of combining these risk factors (AUC $=0.950$ ) was also significantly higher than that of each risk group alone, outperforming previous risk models. 
Table 3 Factors associated with the death of COVID-19 patients

\begin{tabular}{|c|c|c|c|c|}
\hline \multirow[t]{2}{*}{ Indicators } & \multicolumn{2}{|c|}{ Univariable Cox regression } & \multicolumn{2}{|c|}{ Multivariable Cox regression } \\
\hline & $\mathrm{HR}(95 \% \mathrm{Cl})$ & $P$ value & $\mathrm{HR}(95 \% \mathrm{Cl})$ & $P$ value \\
\hline \multicolumn{5}{|l|}{ Vital signs } \\
\hline Temperature, ${ }^{\circ} \mathrm{C}$ & 1.340(1.169-1.536) & $<0.0001^{*}$ & $1.332(1.160-1.529)$ & $<0.0001^{*}$ \\
\hline Heart rate, bpm & $1.036(1.031-1.042)$ & $<0.0001^{*}$ & $1.037(1.032-1.043)$ & $<0.0001^{*}$ \\
\hline Respiratory rate, bpm & $1.118(1.105-1.130)$ & $<0.0001^{*}$ & 1.123(1.109-1.136) & $<0.0001^{*}$ \\
\hline Mean arterial pressure, $\mathrm{mmHg}$ & $0.958(0.951-0.966)$ & $<0.0001^{*}$ & $0.958(0.950-0.966)$ & $<0.0001^{*}$ \\
\hline \multicolumn{5}{|l|}{ Laboratory examinations } \\
\hline \multicolumn{5}{|l|}{ Blood routine } \\
\hline Leukocytes, \# $(N=993), \times 10^{9} / \mathrm{L}$ & $1.075(1.065-1.085)$ & $<0.0001^{*}$ & 1.095(1.081-1.109) & $<0.0001^{*}$ \\
\hline Monocytes, \% $(N=993)$ & $0.822(0.793-0.852)$ & $<0.0001^{*}$ & $0.816(0.787-0.847)$ & $<0.0001^{*}$ \\
\hline Neutrophils, \% $(N=993)$ & $1.106(1.091-1.120)$ & $<0.0001^{*}$ & 1.107(1.092-1.121) & $<0.0001^{*}$ \\
\hline Eosinophils, \% (N=993) & $0.298(0.224-0.395)$ & $<0.0001^{*}$ & $0.301(0.227-0.398)$ & $<0.0001^{*}$ \\
\hline Hemoglobin ( $N=993), g / L$ & 0.994(0.989-1.000) & $0.044^{*}$ & 0.993(0.987-0.998) & $<0.0001^{*}$ \\
\hline \multicolumn{5}{|l|}{ Immune cells subsets } \\
\hline Lymphocytes, \# $(N=993), \times 10^{9} / \mathrm{L}$ & $0.216(0.158-0.297)$ & $<0.0001^{*}$ & $0.220(0.161-0.302)$ & $<0.0001^{*}$ \\
\hline $\mathrm{CD}^{+}{ }^{+} \mathrm{CD} 9^{-} \mathrm{T}$ cells, $\#(N=207), / \mu \mathrm{L}$ & 0.996(0.995-0.997) & $<0.0001^{*}$ & $0.996(0.995-0.997)$ & $<0.0001^{*}$ \\
\hline $\mathrm{CD}^{+}{ }^{+} \mathrm{CD} 4^{+} \mathrm{T}$ cells, $\#(N=207), / \mu \mathrm{L}$ & $1.000(0.999-1.001)$ & 0.786 & $1.000(0.999-1.001)$ & 0.861 \\
\hline $\mathrm{CD}^{+}{ }^{+} \mathrm{CD}^{+} \mathrm{T}$ cells, $\#(N=207), / \mu \mathrm{L}$ & 0.989(0.986-0.992) & $<0.0001^{*}$ & $0.989(0.985-0.992)$ & $<0.0001^{*}$ \\
\hline $\mathrm{CD}^{-}{ }^{-} \mathrm{CD}_{19}{ }^{+} \mathrm{B}$ cells, $\#(N=206), / \mu \mathrm{L}$ & 0.994(0.990-0.997) & $0.001^{*}$ & $0.994(0.990-0.998)$ & $0.002^{*}$ \\
\hline $\mathrm{CD}^{-}{ }^{-} \mathrm{CD} 16^{+} \mathrm{CD}^{2} 6^{+} \mathrm{NK}$ cells, $\#(N=207), / \mu \mathrm{L}$ & $0.986(0.981-0.990)$ & $<0.0001^{*}$ & $0.985(0.981-0.990)$ & $<0.0001^{*}$ \\
\hline T cells $+B$ cells + NK cells, \# $(N=207), / \mu \mathrm{L}$ & 0.997(0.997-0.998) & $<0.0001^{*}$ & 0.997(0.996-0.998) & $<0.0001^{*}$ \\
\hline \multicolumn{5}{|l|}{ Inflammatory cytokines and biomarkers } \\
\hline $\mathrm{IL}-6(N=796), \mathrm{pg} / \mathrm{mL}$ & $1.001(1.001-1.002)$ & $<0.0001^{*}$ & $1.001(1.001-1.002)$ & $<0.0001^{*}$ \\
\hline $\mathrm{IL}-10(\mathrm{~N}=778), \mathrm{pg} / \mathrm{mL}$ & $1.008(1.006-1.010)$ & $<0.0001^{*}$ & $1.009(1.006-1.012)$ & $<0.0001^{*}$ \\
\hline$\| \mathrm{L}-8(\mathrm{~N}=779), \mathrm{pg} / \mathrm{mL}$ & $1.000(1.000-1.001)$ & $<0.0001^{*}$ & $1.000(1.000-1.001)$ & $<0.0001^{*}$ \\
\hline TNF-a $(N=786), p g / m L$ & $1.055(1.045-1.066)$ & $<0.0001^{*}$ & $1.059(1.048-1.070)$ & $<0.0001^{*}$ \\
\hline $\mathrm{IL}-1 \beta(N=778), \mathrm{pg} / \mathrm{mL}$ & $1.018(1.005-1.031)$ & $0.007^{*}$ & $1.021(1.007-1.036)$ & $0.004^{*}$ \\
\hline $\mathrm{IL}-2 \mathrm{R}(\mathrm{N}=774), \mathrm{U} / \mathrm{mL}$ & $1.001(1.001-1.001)$ & $<0.0001^{*}$ & $1.001(1.001-1.001)$ & $<0.0001^{*}$ \\
\hline Ferritin $(N=602), \mu \mathrm{g} / \mathrm{L}$ & $1.000(1.000-1.000)$ & $<0.0001^{*}$ & $1.000(1.000-1.000)$ & $<0.0001^{*}$ \\
\hline hs-CRP $(N=980), \mathrm{mg} / \mathrm{L}$ & $1.010(1.008-1.011)$ & $<0.0001^{*}$ & $1.010(1.008-1.011)$ & $<0.0001^{*}$ \\
\hline Procalcitonin $(N=890), \mathrm{ng} / \mathrm{mL}$ & $1.051(1.036-1.066)$ & $<0.0001^{*}$ & $1.051(1.036-1.067)$ & $<0.0001^{*}$ \\
\hline \multicolumn{5}{|l|}{ Organ damage indexes } \\
\hline $\operatorname{ALT}(N=991), U / L$ & $1.001(1.000-1.001)$ & $0.002^{*}$ & $1.001(1.000-1.001)$ & $0.002^{*}$ \\
\hline $\operatorname{AST}(N=991), \mathrm{U} / \mathrm{L}$ & $1.001(1.000-1.001)$ & $<0.0001^{*}$ & $1.001(1.000-1.001)$ & $<0.0001^{*}$ \\
\hline $\mathrm{TBIL}(N=986), \mu \mathrm{mol} / \mathrm{L}$ & $1.006(1.004-1.008)$ & $<0.0001^{*}$ & $1.007(1.005-1.009)$ & $<0.0001^{*}$ \\
\hline ALB $(N=988), g / L$ & 0.875(0.854-0.896) & $<0.0001^{*}$ & $0.874(0.853-0.895)$ & $<0.0001^{*}$ \\
\hline $\mathrm{LDH}(N=950), \mathrm{U} / \mathrm{L}$ & $1.003(1.002-1.003)$ & $<0.0001^{*}$ & $1.003(1.002-1.003)$ & $<0.0001^{*}$ \\
\hline $\operatorname{ALP}(N=952), U / L$ & $1.003(1.002-1.005)$ & $<0.0001^{*}$ & $1.004(1.002-1.005)$ & $<0.0001^{*}$ \\
\hline Amylase $(N=486), U / L$ & $1.002(1.000-1.004)$ & $0.021^{*}$ & $1.002(1.000-1.004)$ & $0.038^{*}$ \\
\hline $\mathrm{eGFR}(\mathrm{N}=987), \mathrm{mL} /\left(\mathrm{min}^{*} 1.73 \mathrm{~m}^{2}\right)$ & $0.983(0.978-0.988)$ & $<0.0001^{*}$ & $0.981(0.976-0.986)$ & $<0.0001^{*}$ \\
\hline Creatinine $(N=988), \mu \mathrm{mol} / \mathrm{L}$ & $1.002(1.002-1.003)$ & $<0.0001^{*}$ & $1.002(1.002-1.003)$ & $<0.0001^{*}$ \\
\hline NT-proBNP $(N=841), \mathrm{pg} / \mathrm{mL}$ & $1.000(1.000-1.000)$ & $<0.0001^{*}$ & $1.000(1.000-1.000)$ & $<0.0001^{*}$ \\
\hline CK-MB $(N=650), \mathrm{U} / \mathrm{L}$ & $1.039(1.031-1.047)$ & $<0.0001^{*}$ & $1.048(1.036-1.060)$ & $<0.0001^{*}$ \\
\hline hs-cTnl $(N=905), \mathrm{pg} / \mathrm{mL}$ & $1.000(1.000-1.000)$ & $<0.0001^{*}$ & $1.000(1.000-1.000)$ & $<0.0001^{*}$ \\
\hline Platelets, \# $(N=988), \times 10^{9} / \mathrm{L}$ & 0.994(0.992-0.995) & $<0.0001^{*}$ & 0.994(0.992-0.995) & $<0.0001^{*}$ \\
\hline $\mathrm{PT}(N=980), \mathrm{s}$ & $1.046(1.036-1.055)$ & $<0.0001^{*}$ & $1.045(1.036-1.055)$ & $<0.0001^{*}$ \\
\hline $\operatorname{APTT}(N=930), s$ & $1.025(1.016-1.034)$ & $<0.0001^{*}$ & $1.025(1.016-1.034)$ & $<0.0001^{*}$ \\
\hline D-Dimer $(N=971), \mu \mathrm{g} / \mathrm{mL}$ & $1.129(1.113-1.144)$ & $<0.0001^{*}$ & $1.131(1.115-1.147)$ & $<0.0001^{*}$ \\
\hline $\operatorname{FDP}(N=765), \mathrm{g} / \mathrm{L}$ & $1.018(1.016-1.020)$ & $<0.0001^{*}$ & $1.018(1.016-1.021)$ & $<0.0001^{*}$ \\
\hline
\end{tabular}


Table 3 (continued)

\begin{tabular}{|c|c|c|c|c|}
\hline \multirow[t]{2}{*}{ Indicators } & \multicolumn{2}{|c|}{ Univariable Cox regression } & \multicolumn{2}{|c|}{ Multivariable Cox regression } \\
\hline & $\mathrm{HR}(95 \% \mathrm{Cl})$ & $P$ value & $\mathrm{HR}(95 \% \mathrm{Cl})$ & $P$ value \\
\hline PTA $(N=980), \%$ & $0.959(0.954-0.964)$ & $<0.0001^{*}$ & $0.957(0.951-0.962)$ & $<0.0001^{*}$ \\
\hline \multicolumn{5}{|l|}{ Metabolism indexes } \\
\hline $\mathrm{K}^{+}(\mathrm{N}=993), \mathrm{mmol} / \mathrm{L}$ & $1.538(1.295-1.826)$ & $<0.0001^{*}$ & $1.540(1.291-1.837)$ & $<0.0001^{*}$ \\
\hline $\mathrm{Ca}^{2+}(\mathrm{N}=992), \mathrm{mmol} / \mathrm{L}$ & $0.025(0.011-0.058)$ & $<0.0001^{*}$ & $0.025(0.010-0.058)$ & $<0.0001^{*}$ \\
\hline \multicolumn{5}{|l|}{ Blood gas characteristics } \\
\hline $\mathrm{PaO}_{2}(\mathrm{~N}=304), \mathrm{mmHg}$ & 0.958(0.949-0.967) & $<0.0001^{*}$ & 0.956(0.947-0.966) & $<0.0001^{*}$ \\
\hline $\mathrm{SaO}_{2}(N=304), \%$ & 0.956(0.949-0.963) & $<0.0001^{*}$ & $0.954(0.947-0.962)$ & $<0.0001^{*}$ \\
\hline $\mathrm{PaCO}_{2}(\mathrm{~N}=304), \mathrm{mmHg}$ & $0.946(0.924-0.970)$ & $<0.0001^{*}$ & $0.950(0.926-0.974)$ & $<0.0001^{*}$ \\
\hline Actual bicarbonate $(N=304), \mathrm{mmol} / \mathrm{L}$ & $0.922(0.887-0.958)$ & $<0.0001^{*}$ & $0.930(0.894-0.967)$ & $<0.0001^{*}$ \\
\hline \multicolumn{5}{|l|}{ Score prediction } \\
\hline SOFA score & $1.340(1.276-1.408)$ & $<0.0001^{*}$ & $1.437(1.348-1.532)$ & $<0.0001^{*}$ \\
\hline qSOFA score & $3.435(2.577-4.580)$ & $<0.0001^{*}$ & $3.471(2.600-4.634)$ & $<0.0001^{*}$ \\
\hline APACHE II score & $1.172(1.137-1.208)$ & $<0.0001^{*}$ & $1.195(1.156-1.234)$ & $<0.0001^{*}$ \\
\hline SIRS score & $1.854(1.567-2.194)$ & $<0.0001^{*}$ & $1.924(1.620-2.286)$ & $<0.0001^{*}$ \\
\hline \multicolumn{5}{|l|}{ Complications } \\
\hline ARDS & $16.051(8.262-31.186)$ & $<0.0001^{*}$ & $16.216(8.341-31.527)$ & $<0.0001^{*}$ \\
\hline Acute cardiac injury & $11.435(8.543-15.307)$ & $<0.0001^{*}$ & $13.023(9.678-17.524)$ & $<0.0001^{*}$ \\
\hline Heart failure & $10.201(7.860-13.238)$ & $<0.0001^{*}$ & $10.722(8.227-13.974)$ & $<0.0001^{*}$ \\
\hline Acute kidney injury & 5.957(4.707-7.539) & $<0.0001^{*}$ & $6.063(4.759-7.724)$ & $<0.0001^{*}$ \\
\hline Hyperkalaemia & $3.999(3.148-5.081)$ & $<0.0001^{*}$ & $4.041(3.166-5.157)$ & $<0.0001^{*}$ \\
\hline Acidosis & $2.658(2.007-3.520)$ & $<0.0001^{*}$ & $2.621(1.965-3.495)$ & $<0.0001^{*}$ \\
\hline Acute liver injury & $4.097(3.114-5.388)$ & $<0.0001^{*}$ & $4.241(3.212-5.599)$ & $<0.0001^{*}$ \\
\hline DIC & $5.682(4.209-7.672)$ & $<0.0001^{*}$ & $5.819(4.281-7.910)$ & $<0.0001^{*}$ \\
\hline
\end{tabular}

${ }^{*} P<0.05$

Our enrolled survivor and non-survivor patients with COVID-19 were statistically matched and excluded the effects of common risk factors, such as age, sex, and underlying comorbidities, on the COVID-19 death, which had been reported to affect COVID-19 mortality $[6,9,17]$. According to the chest CT's of patients, the non-survivors have more deleterious pulmonary invasion. These pulmonary findings provide evidence supporting that the non-survivors are more likely to develop into severe illness. The current study is designed to identify additional potential risk factors and establish a comprehensive evaluation system for the death risk of COVID-19. The SOFA, qSOFA, APACHE II and SIRS scores have been considered as good evaluation predictors for sepsis, septic shock and multi-organ failure $[3,4$, $6-8,16]$. Here, we confirmed that those with increased SOFA, qSOFA, APACHE II or SIRS score had a higher death risk of COVID-19. Additionally, the AUC values predicting the death risk of COVID-19 using SOFA, qSOFA, APACHE II or SIRS score attained 0.697, 0.610, 0.826 and 0.749 respectively, consistent with previous reports revealing that these scores are useful predictors of ICU mortality [18-20]. Strikingly, it was reported that
SOFA and qSOFA scores had greater capacities predicting death in ICU cohort, compared to APACHE II or SIRS score [20, 21]. However, in the current study, compared to SOFA and qSOFA, APACHE II and SIRS scores are better predictors for COVID-19 death risk and the validation of the scores might been affected by aggravated pro-inflammatory responses in fatal patients.

We found that the serum levels of multiple pro-inflammatory cytokines or biomarkers, including IL-6, IL-10, IL- 8 , TNF- $\alpha$, IL-1 $\beta$, IL-2R, ferritin, hs-CRP and procalcitonin were substantially elevated in non-survivors with COVID-19. Moreover, the increase of leukocytes and neutrophils were also observed in non-survivors. Aggravated pro-inflammatory responses that could lead to increased vascular permeability, extensive pulmonary pathology, ultimately mediating respiratory failure and death[22], which was also found to be a useful predictor evaluating COVID-19 mortality (AUC $=0.757$ ).

Defeating SARS-CoV-2 infection requires well-coordinated innate and adaptive immune responses. Impaired adaptive immune responses may cause uncontrolled inflammatory responses, deleterious tissue damage and even death. Recent evidence has demonstrated that in 


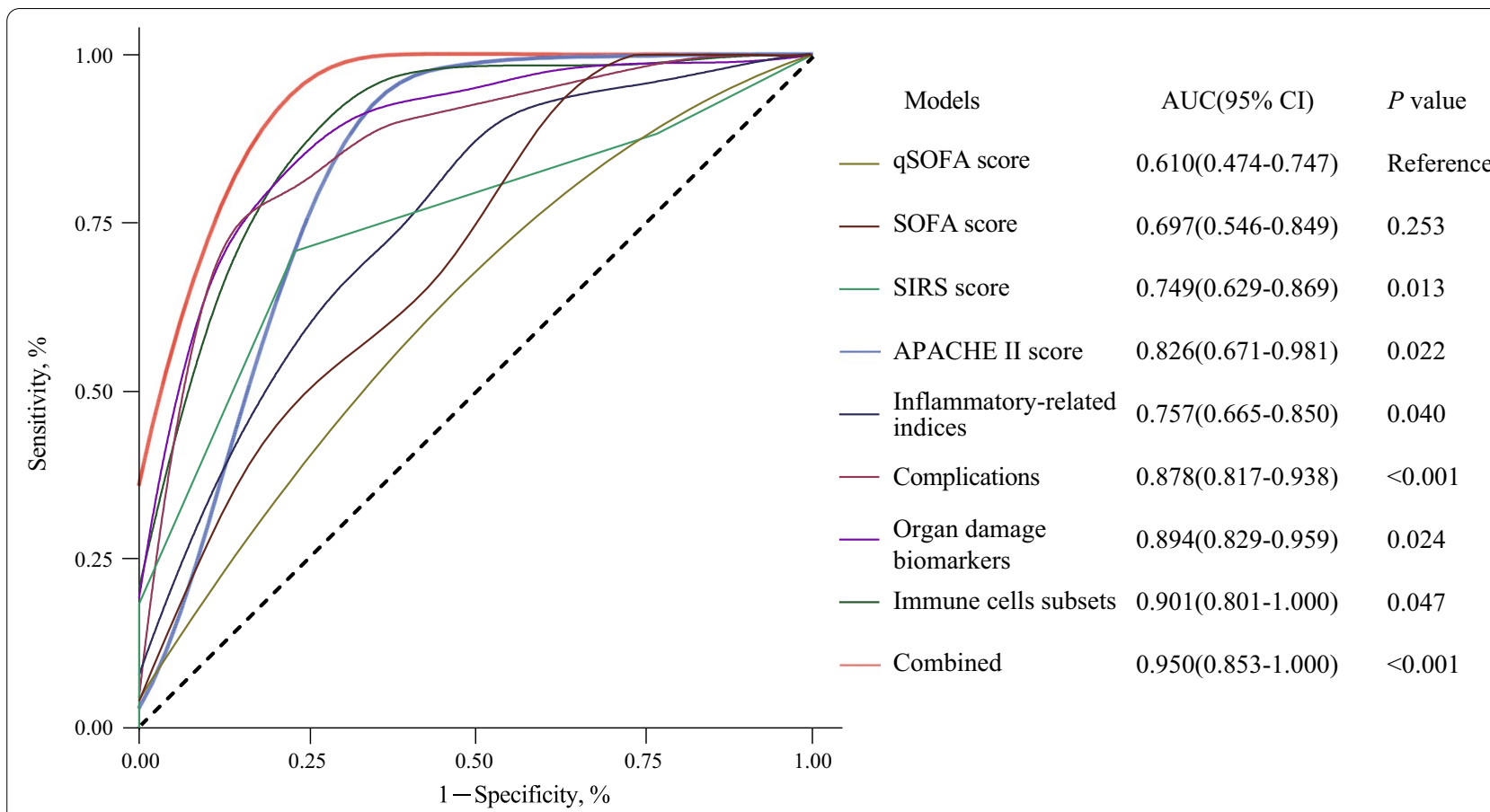

Fig. 2 Comprehensive prediction models for death risk of COVID-19 patients. Time-dependent receiver operating characteristic (ROC) curves and area under the curve (AUC) were employed to assess the predictive accuracy of models evaluating the death risk of COVID-19 with SOFA, qSOFA, APACHE II and SIRS scores, inflammatory-related indexes, complications, organ damage indexes, immune cell subsets and combined group integrating abovementioned these factors. The multivariate Cox proportional hazards model analysis were used to establish a risk model. The stepwise regression was used for the selection of the prediction for the model. ROC curves and AUCs ( $95 \% \mathrm{Cls})$ values were generated to assess prognostic accuracy for each model. A two-sided $P$ value $<0.05$ was considered statistically significant

severe COVID-19 patients, lymphopenia is a common feature, with drastically reduced numbers of $\mathrm{CD} 4^{+} \mathrm{T}$ cells, CD8 ${ }^{+} \mathrm{T}$ cells, B cells and NK cells $[15,23,24]$. These findings were confirmed in our study, revealing that immune cell subsets, such as $\mathrm{CD}^{+} \mathrm{T}$ cells, B cells and NK cells, were drastically reduced in non-survivors, compared to survivors, which is associated with enhanced death risk of COVID-19. Additionally, the AUC value of immune cell subsets that attained 0.901 was highest among risk groups alone. These findings indicated that in death patients with COVID-19, dysregulated immune responses are closely correlated to the poor clinical outcome of COVID-19 and warranted intensively monitor in the clinical treatments.

High levels of pro-inflammatory cytokines and dysregulated immune responses may lead to shock and tissue damage in the heart, liver, kidney and coagulation dysfunction, as well as respiratory failure or multiple organ failure [23]. In the current study, multiple-organ damage biomarkers, such as the substantially elevated concentration of AST, LDH, hs-cTnI, CK-MB, PT-INR, NTproBNP, PT, APTT, PTA, D-dimer and $\mathrm{K}^{+}$, and decreased $\mathrm{Ca}^{2+}, \mathrm{ALB} / \mathrm{GLB}$, eosinophils and platelet counts, were found to be abnormally dysregulated in non-survivors and were significantly associated with increased death risk of COVID-19. Additionally, the patients with lung injury will not only lead to hypoxia, but also inflammatory response, which were more likely to develop complications such as the heart failure, acidosis, acute cardiac, kidney and liver injury, especially ARDS and DIC.. Of note, the COVID-19 patients with complications had a higher risk of developing death, consistent with recent reports which reveal that multiple-organ dysfunction and deleterious complications were more pronounced in severe and fatal COVID-19 cases [5, 9]. Similarly, multiple-organ complications and lung lesions have also been found to be closely associated with poor outcomes in influenza and other respiratory viral infections [25-27]. The organ damage biomarkers $(\mathrm{AUC}=0.894)$ and complications $(\mathrm{AUC}=0.878)$ also presented prominent prediction effects of assessing death risk for COVID-19.

Considering that the indices related to inflammatory responses, adaptive immune responses and multiple organ damage showed great predictive effects of death risk (AUC $=0.950$ ), the allocation of medical resources might be based on these indices. For these areas with more medical resources, inflammatory responses and immune dysfunction control, aggressive supportive 
medical care or earlier admission to the ICU might be the treatment emphasis for patients with high death risk. To date, no vaccine or specific antiviral treatment for COVID-19 has been widely applied, thus supportive therapy that eases the symptoms and protects against important organ damage may be more beneficial for decreasing the fatality rate of COVID-19.

This study has several limitations. First, given that age, sex, and comorbidities, such as diabetes, hypertension, and coronary heart disease (CHD) have been reported as the universal risk factors of COVID-19 death, these factors were statistically matched between survivors and non-survivors and were not included in this study. Our current study was the largest and the laboratory indices were the most comprehensive in fatal COVID-19 patients worldwide. However, due to the nature of the retrospective study, not all laboratory tests were done in all patients, such as lymphocytes subtypes and arterial blood gas tests. Furthermore, this is a single-center observational study, which needs to be further confirmed in additional validation sets.

\section{Conclusions}

In summary, in this large retrospective cohort study with adequate, standardized and unified data, we identified that higher SOFA, qSOFA, APACHE II and SIRS scores, decreased immune cells subsets, elevated inflammation cytokines, dysregulated multi-organ damage biomarkers and deleterious complications were significantly associated with increased death risk of COVID-19. Finally, we established a combined model, comprehensively integrating immune and inflammatory-related factors and other risk factors, which might be more suitable for death risk evaluation of COVID-19 than previous risk models, such as SOFA and qSOFA scores. Our data may provide critical information for the successful clinical management and health decision-making of COVID-19, especially for severe cases.

\footnotetext{
Abbreviations

COVID-19: The coronavirus disease 2019; SARS-CoV-2: Severe acute respiratory syndrome coronavirus 2; SOFA: Sequential organ failure assessment; qSOFA: Quick SOFA; APACHE II: Acute physiology, chronic health evaluation II; SIRS: Systemic inflammatory response syndrome; NT-proBNP: N-terminal pro brain natriuretic peptide; CT: Computed tomographic; ARDS: Acute respiratory distress syndrome; DIC: Disseminated intravascular coagulation; IQR: Interquartile range; SD: Standard deviation; HR: Hazard ratios; Cl: Confidence intervals; ROC: Receiver operating characteristic; AUC: Areas under the curves; IL6: Interleukin 6; TNF-a: Tumor necrosis factor-a; IL2R: Interleukin 2 receptor; CRP: C-reactive protein; ALT: Glutamic-pyruvic transaminase; AST: Glutamicoxalacetic transaminease; TBIL: Total bilirubin; LDH: Lactate dehydrogenase; hs-cTnl: High-sensitivity cardiac troponin; CK-MB: Creatine kinase-MB; ALB/ GLO: Albumin globulin ratio; PT: Prothrombin time; APTT: Activated partial thromboplastin time; ICU: Intensive care unit; IQR: Interquartile range; CHD: Coronary heart disease.
}

\section{Supplementary Information}

The online version contains supplementary material available at https://doi. org/10.1186/s12879-021-06585-8.

Additional file 1. Definitions and references of some abbreviations in the article.

\section{Acknowledgements}

We acknowledge all patients and their families involved in the study and all health-care workers who are fighting against COVID-19 pneumonia. We also thank the assistance of Prof. Gonghong Wei and Prof. Jiping Wang.

\section{Authors' contributions}

$J X$, Ying W, JT, and ZW were the overall principal investigators in this study who conceived the study and obtained financial support, were responsible for the study design, and supervised the entire study. JW, ZX, QZ, XR, CY, XZ, $H L, B L, X C, H X L$ recruited participants. MJ, ZL, XZ, JX, YW, JW and YC drafted the paper. KT, LW, YL, SZ, TD, and XY completed the statistical analyses. HC, JQ, HF, $\mathrm{X}-\mathrm{PY}, \mathrm{ZH}$ and SW completed data analysis, interpreted the results. All authors participated in interpretation data, manuscript writing, and review of the manuscript. All authors read and approved the final manuscript.

\section{Funding}

The publication charge was provided by National Natural Science Foundation of China (Grant No: 81974400). National Natural Science Foundation of China has no role in the design and conduction of the study, sample collection and analysis, interpretation of data, and writing the manuscript.

\section{Availability of data and materials}

The datasets analysed during the current study are available from the corresponding author on reasonable request.

\section{Declarations}

Ethics approval and consent to participate

This study was approved by the Medical Ethics Committee of Tongji Hospital, Tongji Medical College, Huazhong University of Science. (TJ-IRB20200335) Written informed consent was waived due to the rapid emergence of this infectious disease with the permission of the Medical Ethical Committee.

\section{Consent for publication}

Not applicable.

\section{Competing interests}

We declare that we have no conflicts of interest.

\section{Author details}

'Department of Respiratory and Critical Care Medicine, Renmin Hospital of Wuhan University, Wuhan, Hubei, China. ${ }^{2}$ Department of Epidemiology and Biostatistics, Key Laboratory for Environment and Health, School of Public Health, Tongji Medical College, Huazhong University of Sciences and Technology, No. 13, Hangkong Rd, Wuhan 430030, Hubei, China. ${ }^{3}$ Key Laboratory of Environment and Health, Ministry of Education \& Ministry of Environmental Protection, and State Key Laboratory of Environmental Health (Incubating), School of Public Health, Tongji Medical College, Huazhong University of Science and Technology, Wuhan, Hubei, China. ${ }^{4}$ Department of Urology, Tongji Hospital, Tongji Medical College, Huazhong University of Science and Technology, No. 1095, Jiefang Ave, Wuhan 430030, Hubei, China. ${ }^{5}$ nstitute of Reproductive Health, Tongji Medical College, Huazhong University of Science and Technology, Wuhan, Hubei, China. ${ }^{6}$ Department of Critical Care Medicine, Tongji Hospital, Tongji Medical College, Huazhong University of Science and Technology, Wuhan, Hubei, China. ${ }^{7}$ Department of Oncology, Tongji Hospital, Tongji Medical College, Huazhong University of Science and Technology, Wuhan, Hubei, China. ${ }^{8}$ Department of General Surgery, Beijing Hospital, National Center of Gerontology, Institute of Geriatric Medicine, Chinese Academy of Medical Sciences, Beijing, China. ${ }^{9}$ Department of Cardiothoracic Surgery, Nanjing Children's Hospital Affiliated to Nanjing Medical University, Nanjing, Jiangsu, China. ${ }^{10}$ Department of General Surgery, Qinghai University 
Affiliated Hospital, Xining, Qinghai, China. ${ }^{11}$ Computer Center, Tongji Hospital, Tongji Medical College, Huazhong University of Science and Technology, Wuhan, Hubei, China. ${ }^{12}$ Department of Immunology, Tongji Medical College, Huazhong University of Science and Technology, Wuhan, Hubei, China. ${ }^{13}$ Department of Virology, Wuhan Centers for Disease Prevention and Control, Wuhan, Hubei, China. ${ }^{14}$ School of Health Sciences, Wuhan University, Wuhan 430071, China.

Received: 25 July 2020 Accepted: 17 August 2021 Published online: 14 September 2021

\section{References}

1. World Health Organization. Coronavirus disease (COVID-19) outbreak situation. https://www.who.int/emergencies/diseases/novel-coronavirus2019. Accessed 5 Jan 2020

2. Wang Z, Yang B, Li Q, Wen L, Zhang R: Clinical features of 69 cases with coronavirus disease 2019 in Wuhan, China. Clin Infect Dis. 2020.

3. American College of Chest Physicians/Society of Critical Care Medicine Consensus Conference: definitions for sepsis and organ failure and guidelines for the use of innovative therapies in sepsis. Crit Care Med 1992; 20(6):864-74.

4. Knaus WA, Draper EA, Wagner DP, Zimmerman JE. APACHE II: a severity of disease classification system. Crit Care Med. 1985;13(10):818-29.

5. Wang D, Hu B, Hu C, Zhu F, Liu X, Zhang J, Wang B, Xiang H, Cheng Z, Xiong Y, et al. Clinical characteristics of 138 hospitalized patients with 2019 novel coronavirus-infected pneumonia in Wuhan, China. JAMA. 2020:323:1061-9.

6. Zhou F, Yu T, Du R, Fan G, Liu Y, Liu Z, Xiang J, Wang Y, Song B, Gu X, et al. Clinical course and risk factors for mortality of adult inpatients with COVID-19 in Wuhan, China: a retrospective cohort study. Lancet. 2020:395(10229):1054-62

7. Fernando SM, Reardon PM, Rochwerg B, Shapiro NI, Yealy DM, Seely AJE, Perry JJ, Barnaby DP, Murphy K, Tanuseputro P, et al. Sepsis-3 septic shock criteria and associated mortality among infected hospitalized patients assessed by a rapid response team. Chest. 2018;154(2):309-16.

8. Ferreira FL, Bota DP, Bross A, Melot C, Vincent JL. Serial evaluation of the SOFA score to predict outcome in critically ill patients. JAMA. 2001;286(14):1754-8.

9. Chen T, Wu D, Chen H, Yan W, Yang D, Chen G, Ma K, Xu D, Yu H, Wang H, et al. Clinical characteristics of 113 deceased patients with coronavirus disease 2019: retrospective study. BMJ. 2020;368:m1091.

10. Wu C, Chen X, Cai Y, Xia J, Zhou X, Xu S, Huang H, Zhang L, Zhou X, Du C, et al. Risk factors associated with acute respiratory distress syndrome and death in patients with coronavirus disease 2019 pneumonia in Wuhan, China. JAMA Intern Med. 2020;180:934-43.

11. Du Y, Tu L, Zhu P, Mu M, Wang R, Yang P, Wang X, Hu C, Ping R, Hu P, et al Clinical features of 85 fatal cases of COVID-19 from Wuhan: a retrospective observational study. Am J Respir Crit Care Med. 2020;201:1372-9.

12. Veselka J, Faber L, Liebregts M, Cooper R, Januska J, Kashtanov M, Dabrowski M, Hansen PR, Seggewiss H, Hansvenclova E, et al. Short- and long-term outcomes of alcohol septal ablation for hypertrophic obstructive cardiomyopathy in patients with mild left ventricular hypertrophy: a propensity score matching analysis. Eur Heart J. 2019;40(21):1681-7.
13. Tian J, Yuan X, Xiao J, Zhong Q, Yang C, Liu B, Cai Y, Lu Z, Wang J, Wang $Y$, et al. Clinical characteristics and risk factors associated with COVID-19 disease severity in patients with cancer in Wuhan, China: a multicentre, retrospective, cohort study. Lancet Oncol. 2020;21(7):893-903.

14. Heagerty PJ, Lumley T, Pepe MS. Time-dependent ROC curves for censored survival data and a diagnostic marker. Biometrics. 2000:56(2):337-44.

15. Qin C, Zhou L, Hu Z, Zhang S, Yang S, Tao Y, Xie C, Ma K, Shang K, Wang W, et al. Dysregulation of immune response in patients with COVID-19 in Wuhan, China. Clin Infect Dis. 2020;71:762

16. Singer M, Deutschman CS, Seymour CW, Shankar-Hari M, Annane D, Bauer M, Bellomo R, Bernard GR, Chiche JD, Coopersmith CM, et al. The third international consensus definitions for sepsis and septic shock (sepsis-3). JAMA. 2016;315(8):801-10.

17. Onder G, Rezza G, Brusaferro S. Case-fatality rate and characteristics of patients dying in relation to COVID-19 in Italy. JAMA. 2020;323:1775-6.

18. Auriemma CL, Zhuo H, Delucchi K, Deiss T, Liu T, Jauregui A, Ke S, Vessel K, Lippi M, Seeley E, et al. Acute respiratory distress syndrome-attributable mortality in critically ill patients with sepsis. Intensive Care Med. 2020;1:1.

19. Rudd KE, Seymour CW, Aluisio AR, Augustin ME, Bagenda DS, Beane A, Byiringiro JC, Chang CH, Colas LN, Day NPJ, et al. Association of the quick sequential (sepsis-related) organ failure assessment (qSOFA) score with excess hospital mortality in adults with suspected infection in low- and middle-income countries. JAMA. 2018;319(21):2202-11.

20. Raith EP, Udy AA, Bailey M, McGloughlin S, Maclsaac C, Bellomo R, Pilcher DV. Australian, New Zealand Intensive Care Society Centre for O, Resource E: Prognostic Accuracy of the SOFA Score, SIRS Criteria, and qSOFA score for in-hospital mortality among adults with suspected infection admitted to the intensive care unit. JAMA. 2017;317(3):290-300.

21. Fernando SM, Tran A, Taljaard M, Cheng W, Perry JJ. Prognostic accuracy of the quick sequential organ failure assessment for mortality in patients with suspected infection. Ann Intern Med. 2018;169(4):264-5.

22. Prompetchara E, Ketloy C, Palaga T. Immune responses in COVID-19 and potential vaccines: lessons learned from SARS and MERS epidemic. Asian Pac J Allergy Immunol. 2020;38(1):1-9.

23. Cao X. COVID-19: immunopathology and its implications for therapy. Nat Rev Immunol. 2020;20:369-270.

24. Xu Z, Shi L, Wang Y, Zhang J, Huang L, Zhang C, Liu S, Zhao P, Liu H, Zhu L, et al. Pathological findings of COVID-19 associated with acute respiratory distress syndrome. Lancet Respir Med. 2020;8(4):420-2.

25. Corrales-Medina VF, Musher DM, Shachkina S, Chirinos JA. Acute pneumonia and the cardiovascular system. Lancet. 2013:381(9865):496-505.

26. Udell JA, Zawi R, Bhatt DL, Keshtkar-Jahromi M, Gaughran F, Phrommintikul A, Ciszewski A, Vakili H, Hoffman EB, Farkouh ME, et al. Association between influenza vaccination and cardiovascular outcomes in high-risk patients: a meta-analysis. JAMA. 2013;310(16):1711-20.

27. Blackburn R, Zhao H, Pebody R, Hayward A, Warren-Gash C. Laboratoryconfirmed respiratory infections as predictors of hospital admission for myocardial infarction and stroke: time-series analysis of English data for 2004-2015. Clin Infect Dis. 2018;67(1):8-17.

\section{Publisher's Note}

Springer Nature remains neutral with regard to jurisdictional claims in published maps and institutional affiliations. 